\title{
Development of consequence models for two categories of fire through artificial neural networks
}

\section{Supporting information}

Yue Sun a b, Jingyao Wang a ${ }^{\text {b }}$, Wen Zhu a b, Shuai Yuan a b, Yizhi Hong a ${ }^{\text {a }}$, M. Sam Mannan a b and Benjamin Wilhite ${ }^{a}{ }^{*}$

a Artie McFerrin Department of Chemical Engineering, Texas A\&M University, College Station, Texas, 77843, United States

b Mary Kay O’Conner Process Safety Center, Texas A\&M University, College Station, Texas, 77843, United States

* Corresponding author

benjaminwilhite@tamu.edu

3122 TAMU, Room 226

College Station, TX 77843

United States

\section{Table of Contents}

Table S1. Safe related properties of 35 flammable chemicals. ..............................................2

Table S2. Summary of important process condition ranges. .................................................3

Table S3. Parameter specifications of release scenarios in PHAST simulations.......................4

Figure S1. Neural network training state performance for jet fire. (a) $1^{\text {st }}$ model (b) $2^{\text {nd }} \operatorname{model}(\mathrm{c})$ $3^{\text {rd }}$ model (d) $4^{\text {th }}$ model.

Figure S2. Neural network training state performance for early pool fire. (a) $1^{\text {st }}$ model (b) $2^{\text {nd }}$ model.

Figure S3. Error histogram of model performance for early pool fire. (a) $1^{\text {st }}$ model (b) $2^{\text {nd }}$ model.

Figure S4. Neural network training state performance for late pool fire. (a) $1^{\text {st }}$ model (b) $2^{\text {nd }}$ model...

Figure S5. Error histogram of model performance for late pool fire. (a) $1^{\text {st }}$ model (b) $2^{\text {nd }}$ model.

(a)

MATLAB function for jet fire ANN model...............................................................10

MATLAB function for early pool fire ANN model ......................................................17

MATLAB function for late pool fire ANN model...........................................................21 
Table S1. Safe related properties of 35 flammable chemicals ${ }^{1}$.

\begin{tabular}{|c|c|c|c|c|c|c|c|}
\hline Chemicals & $\begin{array}{l}\text { - Heat of } \\
\text { combustion } \\
(\mathrm{kJ} / \mathrm{kg})\end{array}$ & $\begin{array}{l}\text { Auto- } \\
\text { ignition } \\
\text { temp. }(\mathrm{K})\end{array}$ & $\begin{array}{l}\text { Flash } \\
\text { point } \\
(\mathrm{K})\end{array}$ & $\begin{array}{l}\text { NFPA } \\
\text { fire } \\
\text { rating }\end{array}$ & $\begin{array}{l}\text { LFL } \\
(\%)\end{array}$ & $\begin{array}{l}\text { UFL } \\
(\%)\end{array}$ & $\begin{array}{l}\text { Burning } \\
\text { rate } \\
(\mathrm{mm} / \mathrm{min})\end{array}$ \\
\hline Acetone & 30745.52 & 738.00 & 253.00 & 3 & 2.6 & 12.8 & 3.9 \\
\hline Acrolein & 29000.00 & 507.00 & 255.40 & 3 & 2.8 & 31.0 & 3.8 \\
\hline Acrylonitrile & 33200.00 & 754.30 & 272.59 & 3 & 3.0 & 17.0 & 2.0 \\
\hline Ammonia & 22478.57 & 924.00 & 923.70 & 3 & 15.0 & 28.0 & 1.0 \\
\hline Benzene & 40600.00 & 864.80 & 262.00 & 3 & 1.2 & 7.8 & 6.0 \\
\hline Benzyl chloride & 28000.00 & 900.40 & 347.00 & 2 & 1.1 & 14.0 & 4.2 \\
\hline 1,3-Butadiene & 44200.00 & 693.20 & 313.70 & 4 & 2.0 & 12.0 & 8.6 \\
\hline N-Butane & 0.00 & 678.15 & 213.00 & 4 & 1.8 & 8.4 & 8.0 \\
\hline N-Butanol & 2.67 & 618.15 & 302.00 & 3 & 1.7 & 12.0 & 3.2 \\
\hline N-Butyl Ac & .25 & 552.00 & 313.70 & 2 & 1.5 & 9.9 & 4.7 \\
\hline N-Octane & 44426.00 & 493.15 & 286.48 & 3 & 1.0 & 7.0 & 5.2 \\
\hline 1-Butene & 48460.17 & 193.15 & 658.15 & 4 & 1.6 & 10.0 & 2.6 \\
\hline Carbon Disulfide & 13520.00 & 243.15 & 373.15 & 4 & 1.3 & 50.0 & 2.7 \\
\hline Ethane & 47202.00 & 777.59 & 138.15 & 4 & 2.9 & 13.0 & 7.3 \\
\hline Ethanol & 29752.55 & 639.15 & 289.76 & 3 & 3.7 & 19.0 & 3.9 \\
\hline Ethyl & .00 & 763.15 & 137.00 & 4 & 2.7 & 36.0 & 7.4 \\
\hline Ethyle & 8.13 & 702.00 & 253.00 & 4 & 3.6 & 100.0 & 3.5 \\
\hline N-heptane & 44566.00 & 496.10 & 269.10 & 3 & 1.1 & 6.7 & 6.8 \\
\hline N-hexane & 44752.00 & 498.15 & 247.20 & 3 & 1.2 & 7.4 & 7.3 \\
\hline Hydrazine & 19400.00 & 297.15 & 310.85 & 4 & 4.7 & 98.0 & 1.0 \\
\hline Hydrogen & 141800.00 & 773.15 & 20.15 & 4 & 4.0 & 75.0 & 9.9 \\
\hline Hydrogen Cy & 17094.89 & 811.00 & 255.30 & 4 & 5.6 & 40.0 & 1.8 \\
\hline Hydrogen Sulfide & 15240.00 & 505.00 & 190.80 & 4 & 4.0 & 44.0 & 2.3 \\
\hline Isobutanol & 32959.00 & 688.00 & 301.00 & 3 & 1.7 & 12.0 & 3.5 \\
\hline Isop & 7.00 & 672.00 & 284.80 & 3 & 2.2 & 12.0 & 2.3 \\
\hline Methane & 0.00 & 810.00 & 85.10 & 4 & 5.0 & 15.0 & 12.5 \\
\hline Methanol & 22700.00 & 743.00 & 285.00 & 3 & 6.7 & 36.0 & 1.7 \\
\hline N-Nonane & 44321.21 & 478.10 & 304.10 & 3 & 0.9 & 2.9 & 5.8 \\
\hline N-Pentane & 45012.00 & 533.15 & 223.70 & 4 & 1.4 & 8.3 & 8.6 \\
\hline Phenol & 31170.00 & 988.15 & 358.15 & 2 & 1.7 & 8.6 & 3.5 \\
\hline Propane & 46013.00 & 723.15 & 168.70 & 4 & 2.1 & 9.5 & 8.2 \\
\hline Propylene & 45804.00 & 728.15 & 165.30 & 4 & 2.0 & 11.0 & 8.0 \\
\hline 1,2-propylene oxide & 30230.00 & 738.15 & 235.92 & 4 & 2.1 & 37.0 & 3.3 \\
\hline Tolue & 40550.00 & 753.15 & 258.93 & 3 & 1.3 & 7.0 & 5.7 \\
\hline Triethylamine & 39630.00 & 723.15 & 260.93 & 3 & 1.2 & 8.0 & 6.2 \\
\hline
\end{tabular}

\footnotetext{
${ }^{1}$ Source

[1] NIST Chemistry WebBook, https://webbook.nist.gov/chemistry/

[2] CAMEO chemicals, database of hazardous materials, https://cameochemicals.noaa.gov/
} 
Table S2. Summary of important process condition ranges.

\begin{tabular}{lll}
\hline Process & Temperature $\left({ }^{\circ} \mathrm{C}\right)$ & Pressure $(\mathrm{psi})$ \\
\hline Alkylation process & $32-38$ & $\sim 8.4$ \\
Aromatics complex & $185-338,500-800$ & $15-1000$ \\
Adsorptive separation & $130-300$ & $300-700$ \\
DCC & $505-575$ & $10-22$ \\
FCC & $500-550,620-690$ & $14.5-30$ \\
Steam cracking & $760-870$ & 14.5 \\
Fluid catalytic cracking & $700-800$ & $15-50$ \\
Catalytic dehydrogenation & $550-650$ & 15 \\
Oxidative dehydrogenation & $300-600$ & $230-670$ \\
Steam reforming & $815-870$ & $300-400$ \\
Thermal cracking & $400-800$ & $1000-1500$ \\
Selective hydrogenation & $40-100$ & 15 \\
Hydrotreating & $480-670$ & $200-1600$ \\
aromatics hydrogenation & $400-700$ & $500-1400$ \\
Gasoline S Zorb Process & $400-450$ & $250-460$ \\
Gasoline desulfurization & $250-350$ & $200-400$ \\
Delayed coking & $468-524$ & $15-147$ \\
\hline
\end{tabular}


Table S3. Parameter specifications of release scenarios in PHAST simulations.

\begin{tabular}{ll}
\hline Weather category & $1.5 / \mathrm{F}$ \\
Atmospheric temperature $\left({ }^{\circ} \mathrm{C}\right)$ & 20 \\
Surface temperature $\left({ }^{\circ} \mathrm{C}\right)$ & 20 \\
Material quantity $\left(\mathrm{m}^{3}\right)$ & $10,450,4000$ \\
Temperature $\left({ }^{\circ} \mathrm{C}\right)$ & $-50,0,20,100,500,1000$ \\
Pressure (psi) & $1,15,50,500,1000$ \\
Leak hole size (inch) & $2,5,10,25,50$ \\
\hline
\end{tabular}


Figure S1. Neural network training state performance for jet fire. (a) $1^{\text {st }}$ model (b) $2^{\text {nd }}$ model (c) $3^{\text {rd }}$ model $(d) 4^{\text {th }}$ model.

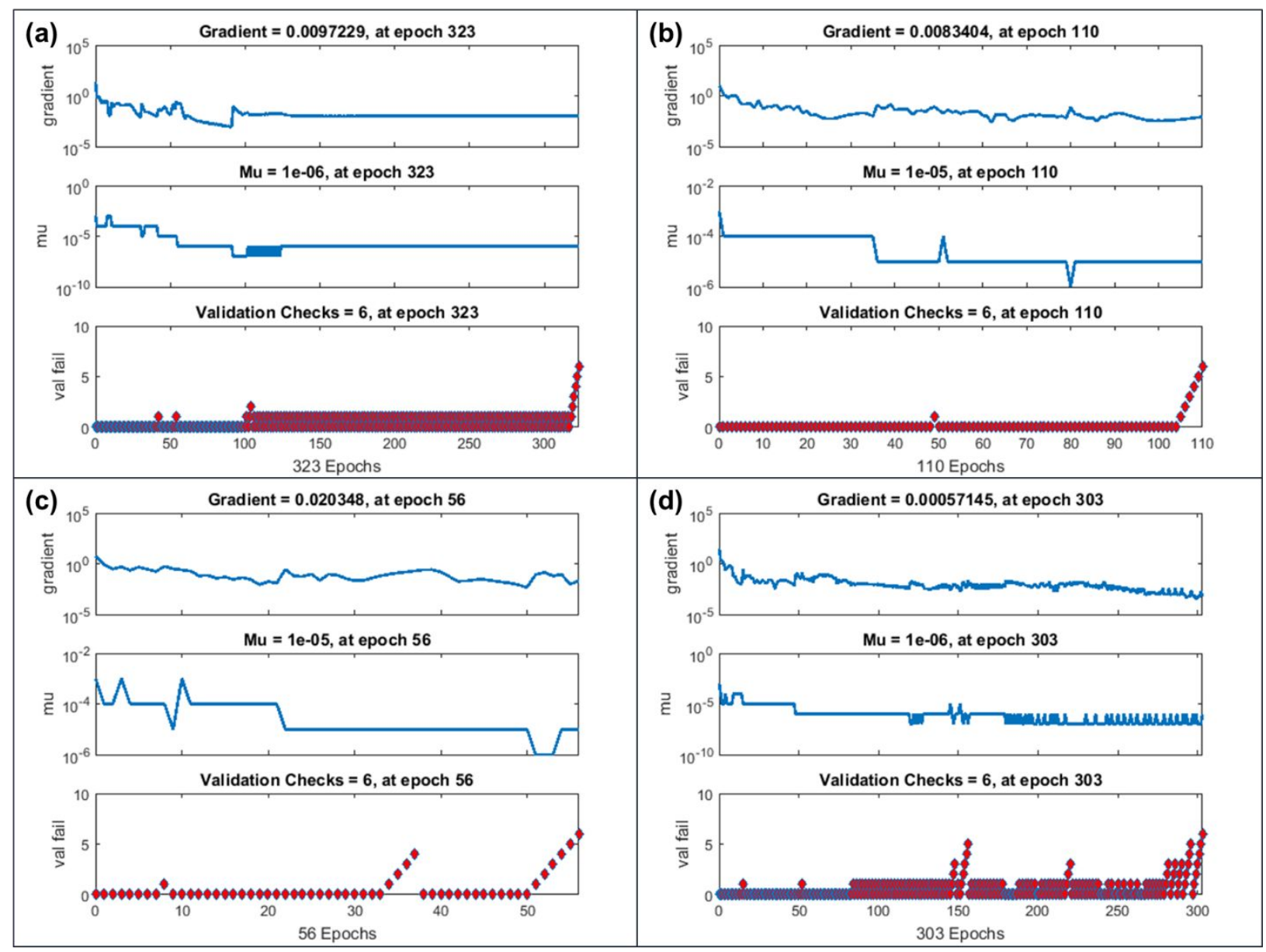


Figure S2. Neural network training state performance for early pool fire. (a) $1^{\text {st }}$ model (b) $2^{\text {nd }}$ model.

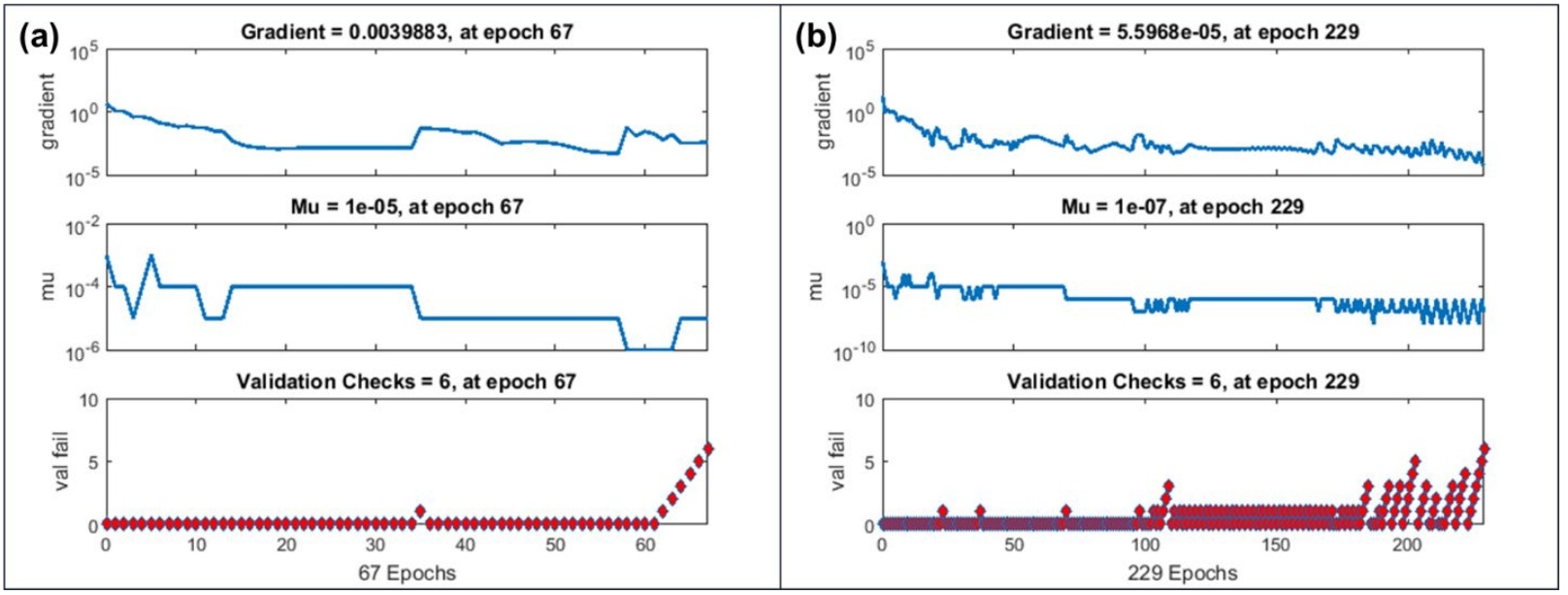


Figure S3. Error histogram of model performance for early pool fire. (a) $1^{\text {st }}$ model (b) $2^{\text {nd }}$ model.

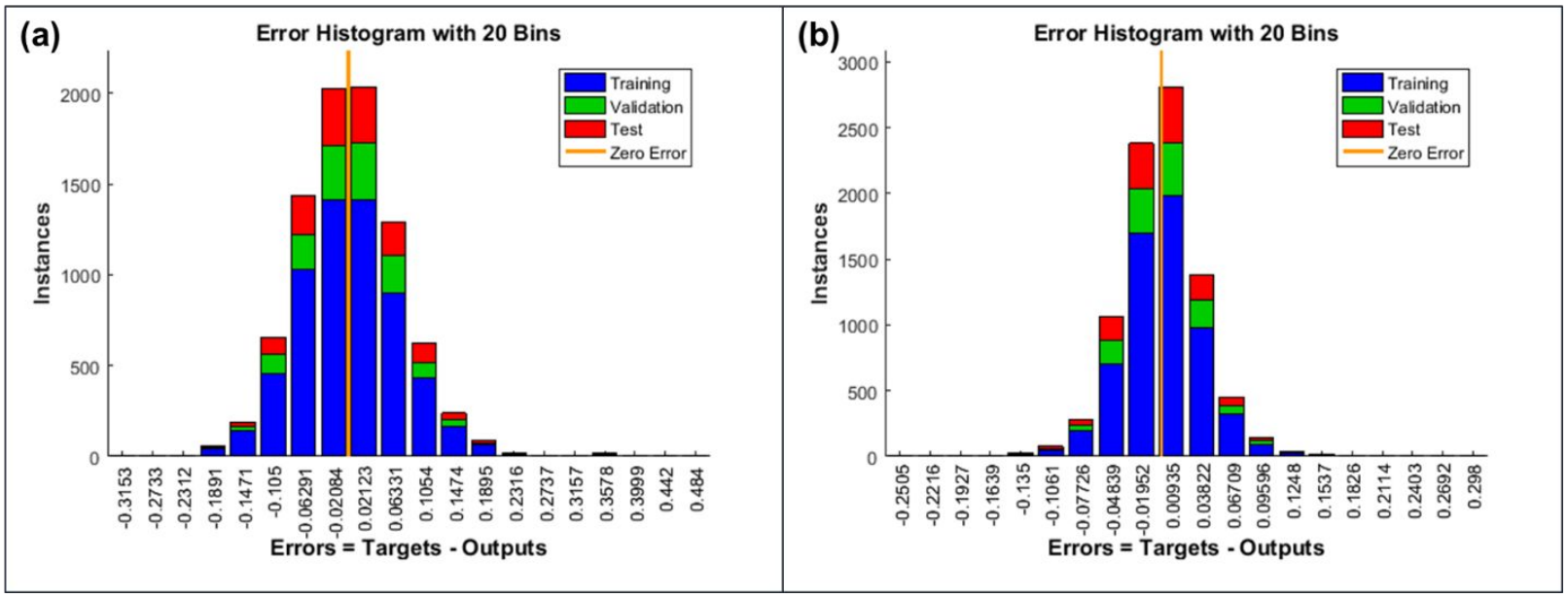


Figure S4. Neural network training state performance for late pool fire. (a) $1^{\text {st }}$ model (b) $2^{\text {nd }}$ model.

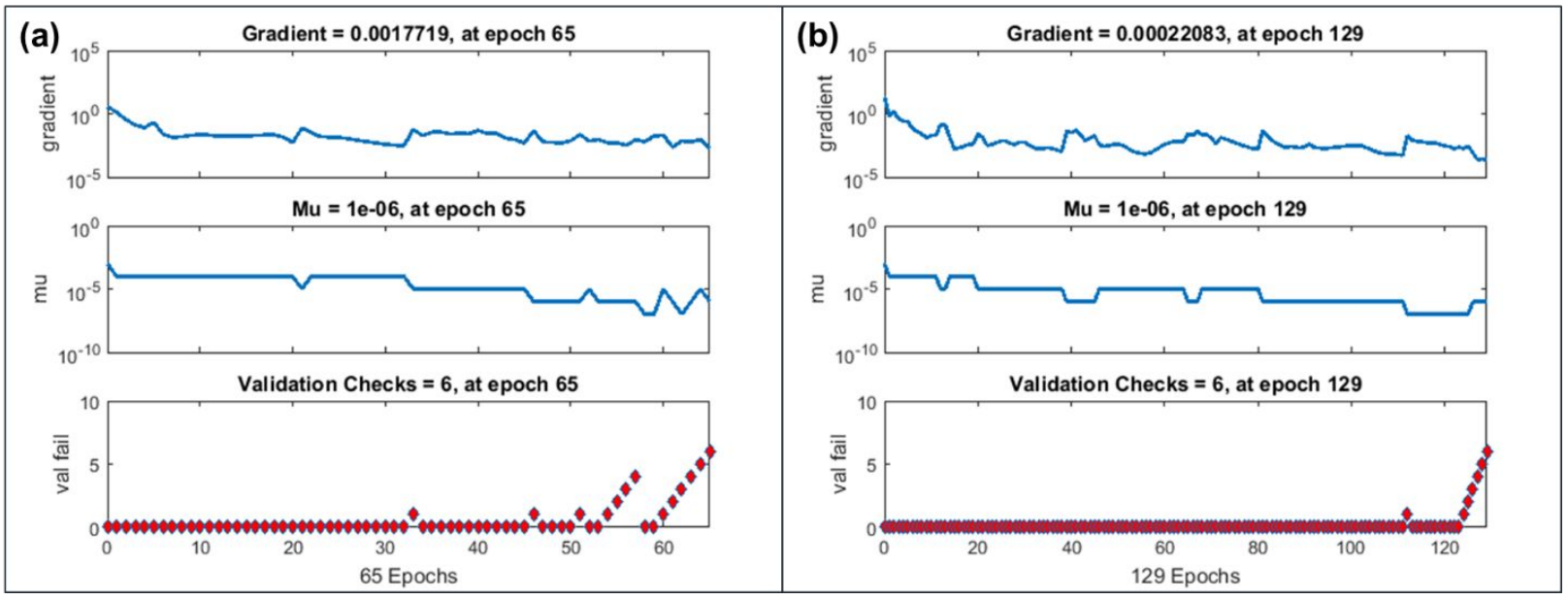


Figure S5. Error histogram of model performance for late pool fire. (a) $1^{\text {st }}$ model (b) $2^{\text {nd }}$ model.

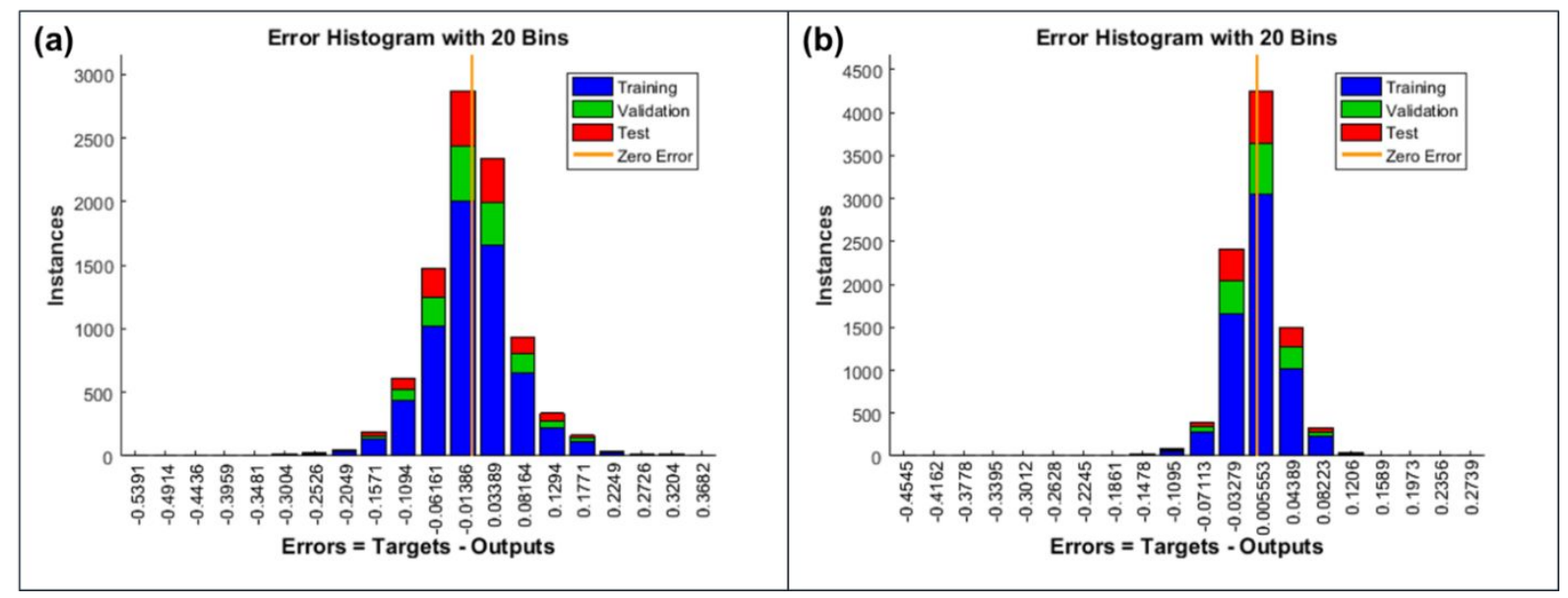




\section{MATLAB function for jet fire ANN model}

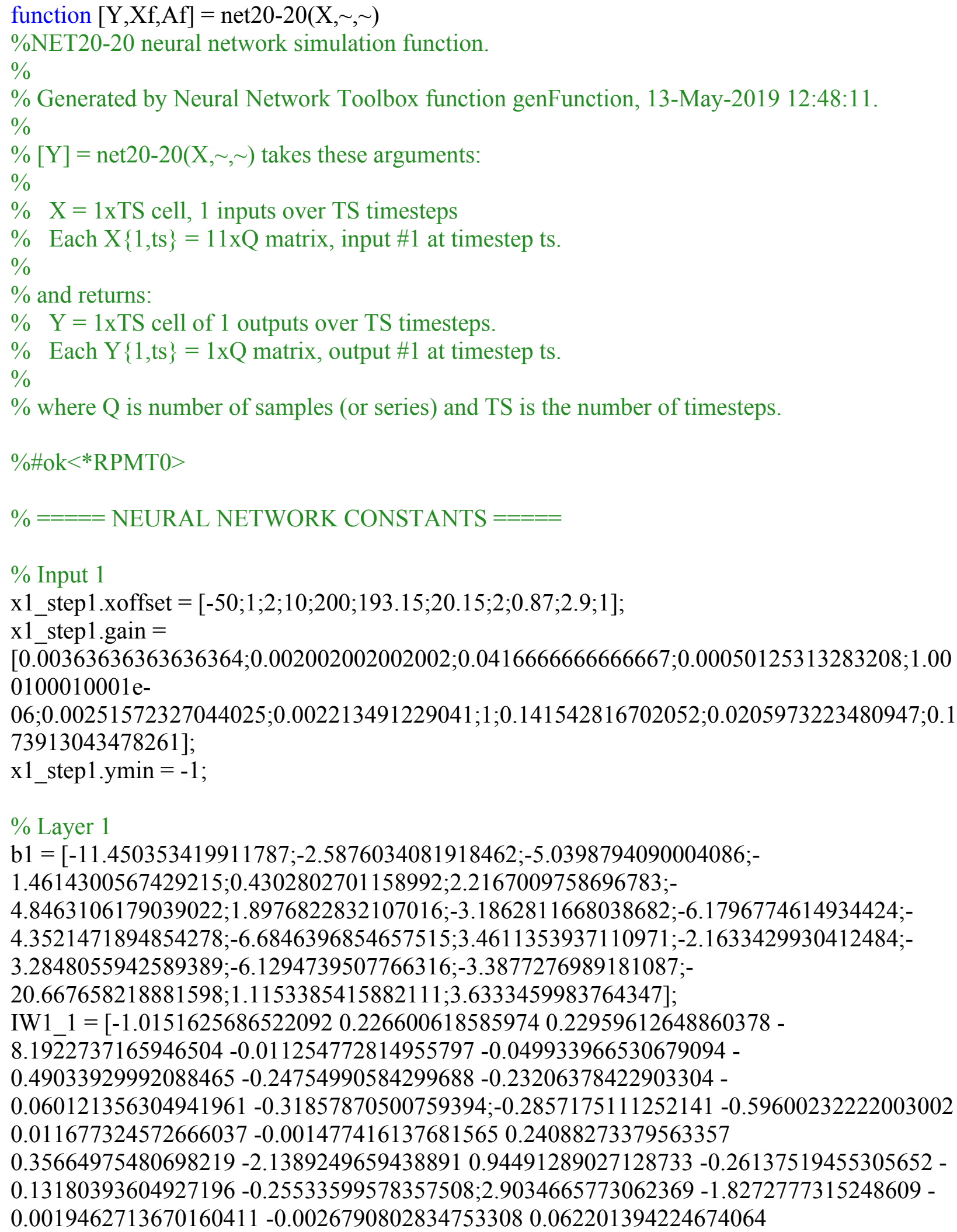


1.6358614801486284 .59405801258296890 .034015247275867647 - 2.3814700793896479 $0.30800375390409857-0.48015103491523148 ; 0.85851655843633912-0.028814938379517742$ $0.15701520421662782-0.00020161237844222536-0.011544786658860799$ $0.025893176022120497-0.00847137439322829910 .0028980323011155934$ $0.320591580373580210 .152483169860341440 .14190859507510739 ; 0.0039743855515911621$ $-2.6695116544505555-0.0317162330938100190 .0011327228076204383$ $0.147426827217313760 .0212764772664773870 .90400983880212504-0.92824466850186427$ $-0.459845157378869551 .59160557872875220 .70491404018843196 ; 1.8435284399728622$ 0.533109621642923750 .0211100400699727390 .0038374341021697009 $0.00471756287943682181 .9375352784613387-2.21836715154961040 .71811818482443246$ $0.176397711584298184 .36024160271007771 .9035786631358675 ; 3.284509107285166$ $1.34732344303598280 .015907190671748252-0.037401282839560769-4.852141090800699$ $10.460963189094731-1.08322249299304851 .2244969154457876-1.1275689328075011$ $2.2172531851052342-1.3990398701124731 ; 0.61483089427017124-0.11536714106575519$ 0.180232390827795490 .00270516094481664880 .036050537146226282 0.0958252108381816290 .0867567921833805040 .044800579573684766 $0.0324573356212839730 .130017191309800970 .14570691459912355 ;-0.68705577495647185$ $-0.0563907945100914770 .017243571803261810 .011854327218474205-1.0420789361129228$ -0.59428898575508082 3.3376866304366799 1.15845466558369650 .86670782209792419 $3.056216989689255-2.4306263816812956 ;-10.3514175000183752 .9471603543460594$ $0.029309183623372658-0.00654045519046500630 .033904639898767665$ -

1.27545174311642780 .492230280645939110 .9526727970283225 .8206399130659436 $5.38260951038139621 .8900929076365995 ; 0.983996295426607380 .0849207869906137$ $0.013155530253819219-0.00127297846082254911 .3931257423581063$ $0.15096902623151179-8.64177797386964210 .0191309252102535720 .8032241934816956$ $3.5286423565790654-1.5946617040913722 ;-0.243786983957565260 .024225589497564429$ $0.0061457699817524004-0.0070449702600972998-5.7437755009569491-$ $0.6233153124339128-6.5452371984033855-0.270344759786696140 .52497139697684525$ $1.13967753995825220 .37684140280874401 ; 4.74659000639279590 .071204087210456818$ $0.0110962153477338880 .00077365558025588332-0.00070992672873454509$ -

$2.4990847226214872 .8125719018277993-0.337431962614105741 .0551959267894699$ $1.73203865664132 .3470901006643774 ;-3.2605778719799527-0.14166009791884068$ $0.0054009045506114642-0.000479136170691331090 .017205920689566463$ $0.451775706395938285 .65611865655327420 .11238990299622019-1.8622031842641402$ $1.1288368582634478-0.96090099743686219 ;-1.43092697601866630 .42800384341731434$ $0.00309847606802094452 .5162639850855148 \mathrm{e}-06-1.8433255753600823$ 4.05156594275035612 .98759036097583010 .930566757345296260 .84775869397714665 $1.0471561834136365-1.5319725952382846 ;-2.15745034150386860 .11326017057360332$ $0.0884653428164105110 .00065490522058199885-0.027419355290243617$ $0.589941784091510990 .27848285571162379-0.136935840505229083 .2294200329024503$ $6.7923460752774911-1.8415699194279422 ; 0.145533517489498690 .13994016422751357$ $0.00010084099982441585-0.969400879147688472 .4326048783302974-3.618257519594839$ $3.4671298134528166-0.281688943775538445 .05675695420347987 .4363825592723733-$ 1.6267100464976574;-0.1543192664136927-21.459808939746868 -0.0085196464515747191 $0.0022609300127153886-0.048224855374125186-0.81509979627575924-$ $0.043202124377638457-1.08734235083700970 .201654909565391670 .086379412646082745$ 
$0.077086894999191372 ; 0.588599182783882460 .10800225210337228$ $0.00346771180965601110 .0014947473650560874-0.0015765022336716814$ $0.15856053127605255-0.0755627081695365440 .025933929467240553$ $0.142539911356803940 .728346617266223070 .26904388656848321 ; 0.014643748538468528$ $0.00866414739391090961 .8817711442692042-0.0020169446379806099$ $0.032557322139315129-0.00902928887684099210 .025258605550584477$ $0.00368690101070610330 .028934136003315312-0.0065749992505792082$ $0.00031860115685143869]$

$\%$ Layer 2 b2 $=[7.5438208364258132 ; 2.5850478977368976 ;-6.016867619590295 ;-$ $14.209737738039234 ; 10.267986238083477 ;-$ $5.267199131461898 ; 0.50851568889576404 ; 12.589285369307817 ;-$ $0.44513389980684143 ; 10.739328744051347 ;-$ 5.5275459590757681;7.6716449361841965;3.8099525196214397;-3.8674950984714598;$0.64642808336599022 ;-5.5489840177172525 ; 19.183010747846772 ; 10.438144007045191$;$1.5060947813662462 ; 9.6206402137724627]$

LW2_1 $=[-0.1853187664349786-0.90014902769854111 .200122189446249$ $0.12 \overline{18} 1441584800022-0.66364756328719743-4.6258685559727661-0.70879724016370993$ $0.62923178603743646-1.4872459600431460 .398962715697990630 .088496608503418256$ $2.40289387917238571 .1945033723715057-0.664961780642776451 .2225908161207331$ $0.0918970610900727718 .7047896219442897-2.57254868886522251 .0862541600189712$ $1.4772973679917558 ;-0.3156408603128058-0.477958136540228450 .51299434309859471$ $0.59798676626452685-0.275461996485786560 .11769928140427785-0.07812148022294757$ $0.862309689068389340 .170353783965141170 .32815302675136554-0.72061175171516789$ $0.928885848762961540 .6214648525049159-0.0680837956221050510 .78770117676657037$ $0.11549460237863485-0.16385934639458810 .8805390697491049-1.4627004837541047$ $0.86330625482039036 ; 6.5560002678141789-0.751603501718763 .3260508394311556$ 4.56421898732900020 .337123208679170233 .55659168940423821 .5897287572940568 $0.77816235763090746-0.811628988715035193 .90762644982091611 .2999879187318562$ $6.12410386634897371 .367101029632023-0.710693038076065434 .4485720513423779$ $7.3644886573809698-7.36659610638662170 .48380411298284526-2.5292859778501189$ $7.0148320726492042 ; 2.1681635924683476-0.279265359520600332 .5059330012419756-$ $2.24388889785049760 .55195377192148198-6.6833129888944578-1.79676371458934$ $0.36151592332380322-1.06201546195995368 .7450978938330763-10.362431150694375$ $6.66344967151052188 .8040759596720974-8.9173066081030861-3.0458081536049866$ $1.5456555018112283-1.1016657150581926-7.672069445582455-8.7188896581703137$ $4.0078043404766275 ; 5.76200342620010993 .0660118734641499-1.5248807594404703$ 0.427858786035242470 .899792825766259070 .0896584290477910470 .28733779785605151 $0.14644062374720468-0.54120253389288109-1.4201302704152696-0.88913821759638312$ $0.958565794394546140 .91035259817874747-0.77883149657994022-0.91988905740925364$ $0.881008286831046152 .37893415599276681 .5844884012076004-3.0009396080533191$ $1.0272094582714173 ;-1.6685464984136469-0.626825904353376930 .7394091834274239$ $1.1533218987045841-0.18436419476479038-1.0986804779879176-0.42724723195633868-$ $0.23826875332746781 .70541769895810250 .84438372421044949-2.3673012369778754-$ $0.367029436694789050 .057483711928399253-0.86345155727608591-0.30146438466962533$ 
$-0.966854473843559380 .74608086376152849-1.624174478756621-3.8314788236340602$ $3.2618305687210563 ; 1.8077164200179126-0.10495645246163732-0.31819966458358379$ $1.0113693401543711-0.269475939048322341 .23665351964329170 .072685439637864377$ $0.28961611441252849-0.58023691970826641-0.44742613911463563-1.1039003000741188-$ $0.54081195859343179-2.17118517684916150 .233230032583898460 .18640996821238151$ $1.5785677174480857-1.04906993335444980 .70948655472571043-0.61807791010471413$ $1.472925820261844 ; 0.905300406334858530 .51414349855756292-0.347537115121829$ $1.0201795107546174-0.42558638883596717-2.034018966999803-0.52584891739147488$ $0.010037860783897724-0.43757156749938769-0.091638461619890255-$

$0.0642620057452597121 .67155633246655771 .1919321647690604-0.47737371921577049$ $1.59627652633369331 .0533651874474190 .9609279872254527-0.32932242411498147$ $0.52906735024587193-4.6119488464975804 ;-1.26655648077461862 .1418356483479655$ $4.9805473234840933-0.64992514403324764-2.559027484752868-8.1553487992950853-$ $0.75752110370908543-0.4054818393431851-5.127067803286411-0.57212853898509353$ 0.949336727595394342 .19262077959657552 .36240497994304693 .3521895750885822 $3.23785095708395641 .5333679385094713 .9921319347660269-6.1374649985365979$ $5.14414684618606352 .4693313839722566 ; 1.0822487258984195-0.23936725772591169$ $2.9276917960858428-9.743239119183734-0.639929561451587190 .041509923701251755$ $0.0842753441759893924 .3247521406107854-0.66907671133181035$ $0.00608130551365420461 .3914749931464836-1.63982557876374954 .8746071789279259$ $0.14841445185125862-0.0230693783393461390 .082701046292483357-2.6867025591821414$ $-0.036536192898843895 .4602867560926054-20.212725694188574 ;-0.9042024267342188-$ $8.27793344190647140 .097629019078225562-3.09528791221456330 .53798952918417597$ $0.65773669192521977-1.2971770086554693-0.0553244085313310613 .1084316817433231$ $8.0638389984516206-6.0566143742685963-2.01782391398963952 .0265008457586799$ $4.1284174837656034-2.4870046965041577-2.32753225244682322 .6016153777681352$ $0.058934663177862404-14.2993588122318072 .2563624865543512 ; 3.6051403749424211$ $0.37538729554027633-0.17138780434231143-0.768644133747282350 .33163102827869051-$ $0.39084424267453716-0.0513638442564653894 .9473954419181325-0.071528855396066862$ 0.180680925406829870 .138265356038580730 .0974463030321770230 .21896146004646672 $0.205162937292425520 .034102069373051463-0.072944878568358323$ 0.197521311054930680 .423657144125731520 .56176712574578103 $9.766183034487268 ; 2.14136845817245011 .4024442286286469-4.6846265205129107$ $0.69882023945761462-0.075745547541543695-1.6941814114197640 .21446246206136649$ $2.4674845855338274-0.74528332503275219-0.261046982687499821 .0647291044717893$ $1.24111775191836384 .96870285546807370 .72404241999295149-0.46193072497404258$ $0.64579039864636756-0.72045233371510997-1.57243377894226163 .4099310429507494$ $0.70250088822001144 ;-2.6122103958710845-0.378017937686628-0.51067615433077285$ $0.80463248370385765-0.273999020981312121 .75329181978849350 .2150744952883053-$ $0.81464031689024929-1.8218452666108418-0.765394288728033610 .96462704984510017$ $2.0710412616411187-2.0545496290365351 .6882414415601754-0.48600705570044195$ $1.8128074627317128-2.3618390749589273-0.477673329253440474 .5994723319370783$ 1.6513964174571714;-6.965531552342930.73089118701255429 -3.6338588203911297 $2.2803989525198203-1.48739891197547780 .412424616217713870 .3778162006381246$ $9.1984943845348219-1.4530320507355416-5.31810366145176690 .95418616773003506$ $1.4279846928870803-1.1457242351617729-1.98533417436408130 .94657195837084396$ 
$0.384281632091859567 .3461114928750408-3.41197069647351951 .8559135582879991$ $2.5210128609098041 ;-1.5781296542177163-0.61286447785745791 .1490309398671885$ $0.22625553343371002-0.16582256471645707-0.66032512012221922-0.32693318322964376$ $-0.415927539738264241 .43644569202395140 .74623564739597248-2.1063400892398922$ $0.480098768418374010 .083288673817903836-0.77036808025659587-0.28622164367617492$ $-0.81725540712259870 .45531912205113073-1.2561462736462683-3.4419892349186525$ $3.202906536842232 ; 2.1003117966762557-0.0635613084943808392 .7258286595695096-$ $0.0799383568095456551 .7759251092557504-13.747335240109669-1.4697469372371337$ $5.7602497347597792-10.8616190146001074 .236512062775905614 .110059430351827$ $19.46031580024122926 .5485456431990840 .67305569922360375-13.807358883239337$ $6.900623922422419712 .2697752662045620 .64982821871697727-10.701654465158285$ $0.24714021269780329 ;-5.1755709711563433-9.1630389864623005-10.536890545500958$ $1.80203616061113330 .461588772818038238 .5014352208257176-2.5445082875356104$ $7.575182759659749213 .083876175014087 .5218799443318156-13.170222287276653$ $12.2296785509374549 .9137346606544092-1.95515197224817348 .7117616725883522$ $7.0756938924562984-8.29211775865935860 .19819223340885761-0.089694220141082651$ $2.3500738069525551 ; 0.85811885443557090 .70948368646460336-0.5790846697660007$ $0.342335466405078170 .30900551408466548-0.0088496416840995341$ $0.0892728890094128951 .3463193324830629-0.085295091668220982-0.52633228721230141$ $0.18883055495118475-0.945242918088834430 .22436771085067719-0.38728229801119235$ $0.952649387965830760 .367058797429498430 .48228454956025157-0.73461864433763691$ $0.18520673275375307-0.84001209190014192 ;-2.0451601287777446-9.9029236525047519$ $4.79990687393200322 .8559695597572992-0.96499768383167506-4.1202775361753918$ $2.7912580674406424-1.719454863135095-10.666267673793524 .6185097263929276$ $3.6678128535943189-1.7518264604878916 .885084773867222726 .235804126503776$ $14.805030943017972-12.547106253578098-0.30371275630748684-10.47673383740401$ $10.98862427302528-0.97823105539551536] ;$

$\%$ Layer 3 b3 = 6.361974227066078;

LW3_2 $=[0.77142531139168602-7.1822004618366426-18.030943303923912$ 0.977421090383550630 .852210896577771946 .85466379942891282 .3622614633330223 $26.347730719427179-0.373461688127162412 .60735457181381850 .46790682485487778$ $3.72187258075070521 .484378789874383-1.5535478898406503-0.32215227034122268$ $9.25280694631285882 .006669128705984622 .958233481129792-6.4875319759902084$ $0.65343760237337578]$;

$\%$ Output 1

y1_step1.ymin $=-1$;

y1_step1.gain $=0.704828493450882$;

y1_step1.xoffset $=0.726581477010775$;

$\%====$ SIMULATION $=======$

\% Format Input Arguments

isCell $\mathrm{X}=$ iscell $(\mathrm{X})$; 


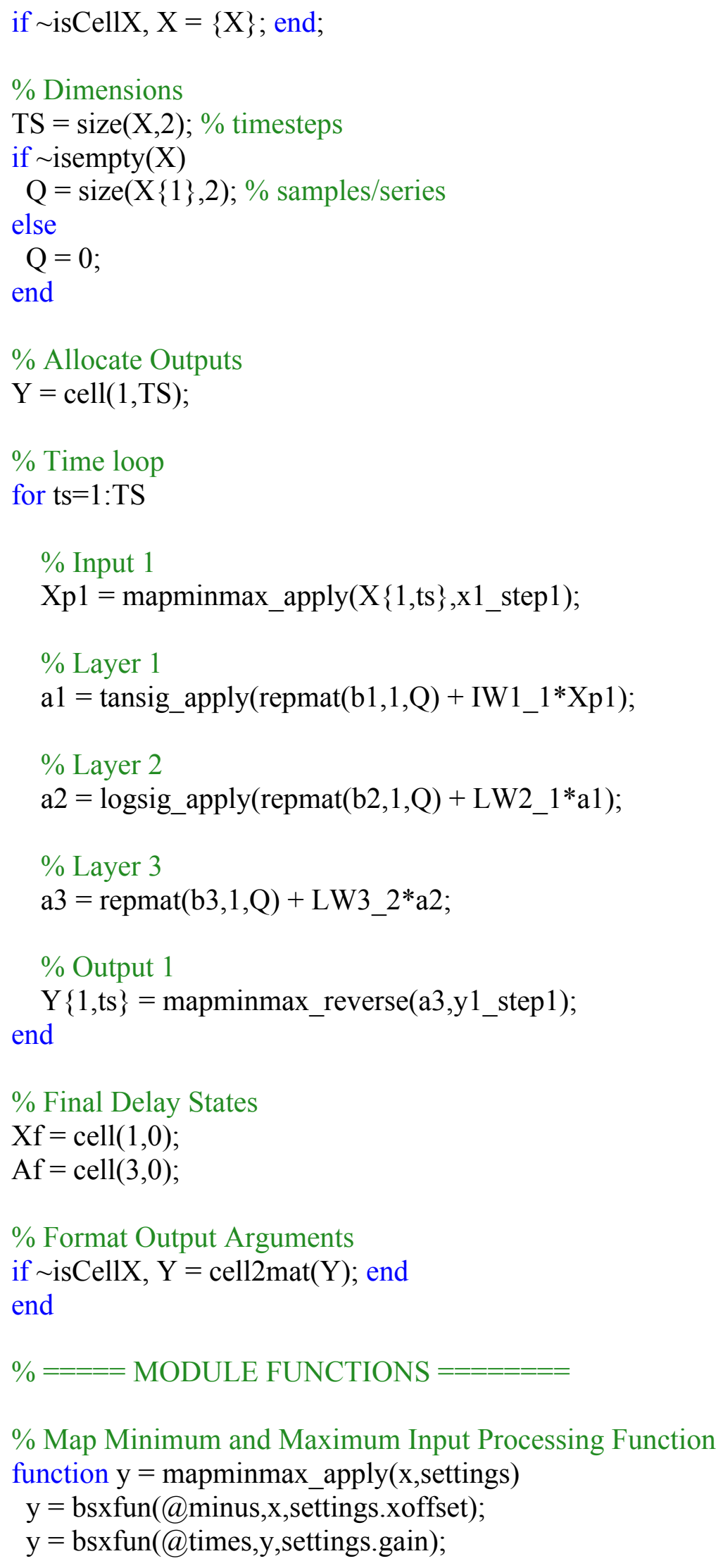


$\mathrm{y}=$ bsxfun(@plus,y,settings.ymin);

end

\% Sigmoid Positive Transfer Function

function $\mathrm{a}=$ logsig_apply $(\mathrm{n}, \sim)$

$\mathrm{a}=1 . /(1+\exp (-n))$

end

\% Sigmoid Symmetric Transfer Function

function $\mathrm{a}=$ tansig_apply $(\mathrm{n}, \sim)$

$\mathrm{a}=2 . /(1+\exp (-2 * \mathrm{n}))-1$

end

\% Map Minimum and Maximum Output Reverse-Processing Function

function $\mathrm{x}=$ mapminmax_reverse(y,settings)

$\mathrm{x}=$ bsxfun(@minus,y,settings.ymin);

$\mathrm{x}=$ bsxfun(@rdivide,x,settings.gain);

$\mathrm{x}=$ bsxfun(@plus,x,settings.xoffset);

end 


\section{MATLAB function for early pool fire ANN model}

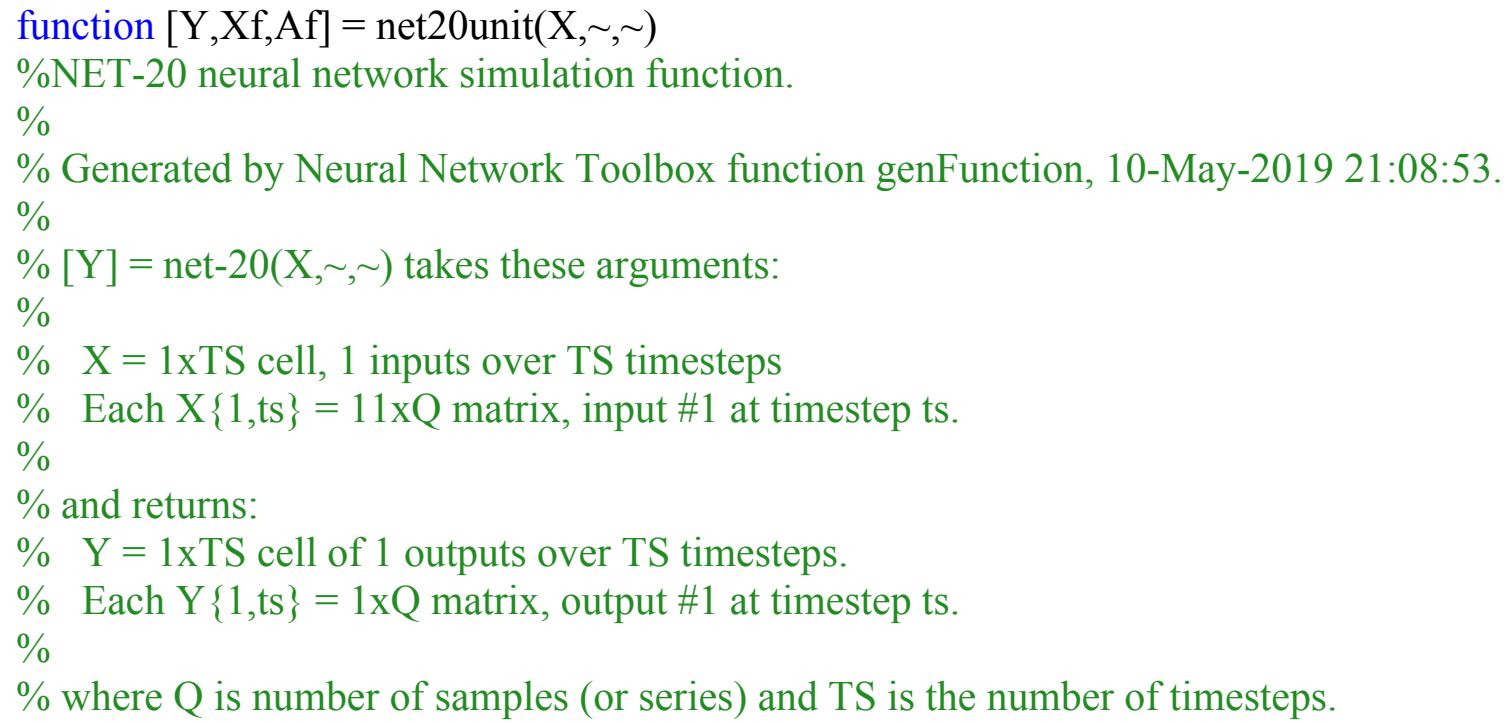


$0.15502419775572723-0.743140141112584642 .2250655111046851-$ $1.4765712672758424 ; 0.0012918760691664241-2.6034039250100407-0.066970527861350229$ $0.0511331374095495080 .062694842418678398-0.068143301172463422$ $0.10345133698568981-0.10538985403954010 .16814538127406745-0.024982745849847384$ $0.21254508423779361 ; 0.01084259996607898-0.0160087215827934663 .3680791204714318$ $0.55821501842407273-0.92358795688972317-0.165410266069054820 .87869611894178457$ $0.037144745208000554-0.087232855652109226-0.0089489263138623765$ $0.24404751346520936 ; 0.0271526763210171930 .682598291381319781 .8400634259754758$ $4.3149736819549567-7.1676415593758254-0.028421632128903709-0.065634574050474556$ $-0.0870941608858091660 .637994030610095410 .056597248193487749-$ $0.095503854532190222 ; 0.240397450628359680 .19177296576287178-2.3418139210573052$ $13.703473983255703-1.9165260470845662-5.107558496725684626 .50844326359098$ $10.133303369691731-9.99561027386043412 .648815268546107-5.4320753600230978$;$0.0019300156408296766-0.39670221326363569-0.0647995501147407983 .967824922797301$ $-2.2087735988446195-7.368662350724053-0.29530799927181051-8.3194581312056037$ $6.9567216905740938-5.92366688462224826 .7637134955695926 ; 0.031045278235975401$ $1.13296022978444521 .623749089280194-1.1104703330272496-10.078178776378618$ $0.16444480295191433-0.3999349032935523-0.0209462486512307891 .0778966385815745$ $0.088520086438766951-0.27165735332565732 ;-0.011352042075565156-$ $0.0435578002987136795 .9525168578975274-3.07591347475772280 .66847626918425118$ $0.10238146844750011-0.097115232132458748-0.155664628177580980 .16231893085951754$ $0.12268911951277331-0.050173797507775529 ; 0.0052544891654514071-$ $0.046199126578726428-0.45275529035212042-4.36118644075235910 .88085335702420231$ $0.157560988256734150 .11121710327528327-0.086650027884176514-0.82239781349703289$ $0.300378521245024950 .19411530454463705 ; 0.0752505195461121710 .050519724563200183$ $-0.1486027171979287215 .1110704288451071 .8898558965376999-13.489466798912838$ $6.18923197723124928 .77775595525751133 .500497809140521-10.600639726462989$ $8.2027511206283403 ; 0.0100865816244279810 .042085381579323206-$ $0.0243385694020095580 .0968480033954520064 .8141156288531386-0.71761088656101202$ $-4.4164795709735749-0.71161663199161884-4.6680844144918272 .3414812701287726$ $0.81329727486823533 ; 0.0068266095717698450 .020163159017829873-$ $0.018302308924034966-0.22448545916809937-13.6457422947592960 .51267721039400327$ $1.8908258596916681-0.81272251189445233-2.19218706428540511 .144579225685028$ $0.085897692666109249 ; 0.0474118048326182540 .040131100819550299-$ $0.0273382337326438690 .023511143615270569-1.5077498994800103-1.5493524556422567$ $1.6894085882650842-1.96799701522860021 .87862112326323311 .5980945462895224$ $0.001353638344443519 ;-0.015726699058318909-0.18940164965999057$ 0.0318568298795713980 .0864820796439056964 .05966332249706060 .44171209351736296 $0.52219871435140341-0.120282274611922942 .7185613527351573-0.087066935298291959$ $0.21468445676466719 ;-0.10300850480252276-0.067149613369154051-$

$0.244436509470702753 .821181524241398-8.08521934912397999 .4666321058235514$ $1.6890323156580542-4.0717203697455115-7.4119113361873175 .1156487054651194$ $2.3635260123647859 ; 0.16771875061059402-1.245428146090126-1.0571764383419355$ $3.7723260070664195-1.62171774430065410 .7889926694813888-8.6152758837078842$ $0.531990638514481929 .288790455461065-0.75507924775104485$ $2.5536576374984166 ; 4.84803466859040630 .88027023853783926-0.18666114649897786$ 
$1.18825065416451468 .4737316129713225-0.126281502356670687 .309348215557506-$ 2.13174886056299334 .1795545122865532 -1.2164873152959059 -2.1140820134764633;$0.692097328835633463 .3724715598595481-11.027570434047082-2.2354456606092068$ $0.787594387516085570 .206649432203825785 .5490926192847327-2.405888430330863$ 8.8319162009099426 0.27369300703242921 4.2756867133452889];

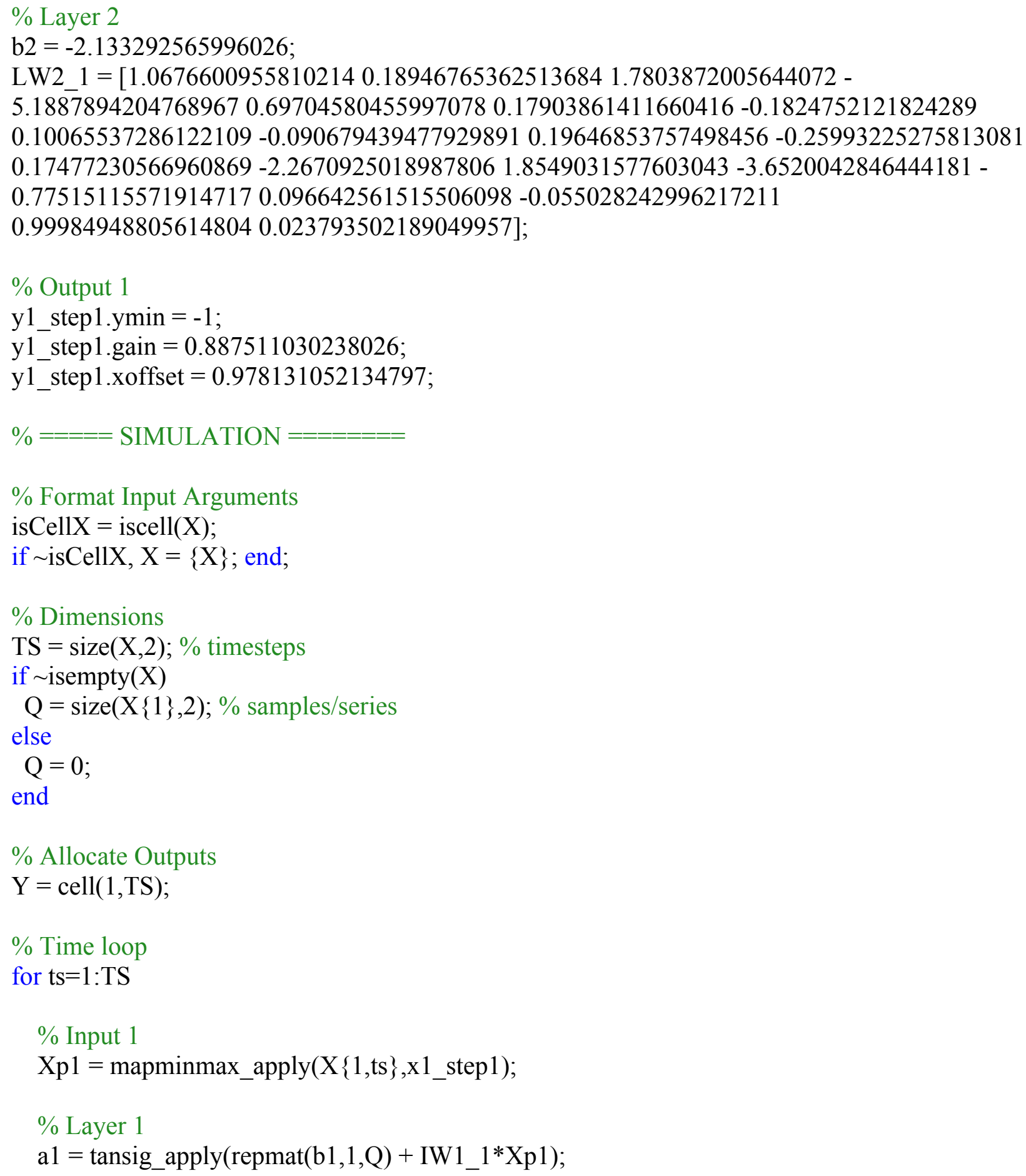




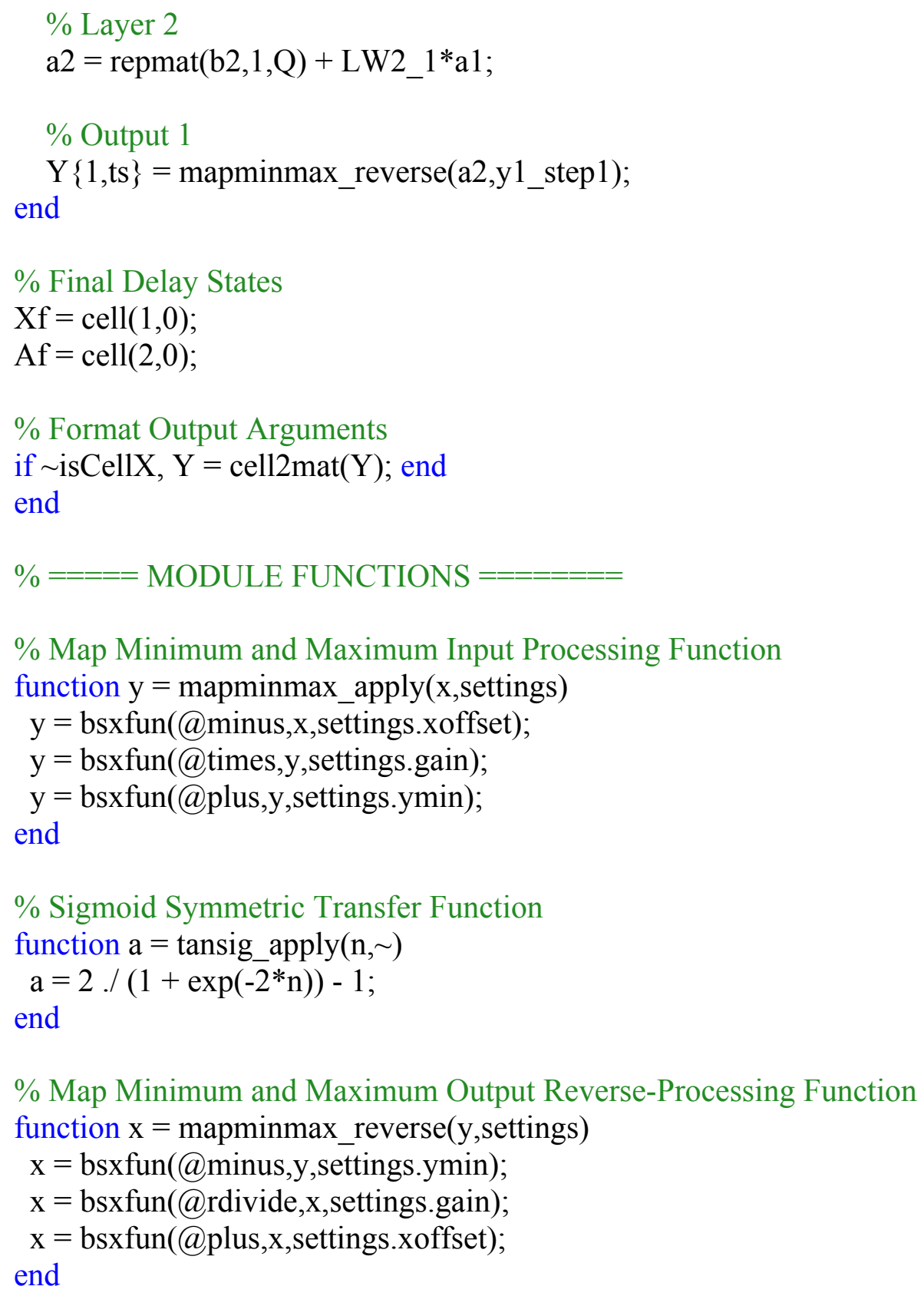




\section{MATLAB function for late pool fire ANN model}

\section{function $[\mathrm{Y}, \mathrm{Xf}, \mathrm{Af}]=$ net20unit $(\mathrm{X}, \sim, \sim)$}

$\%$ NET20UNIT neural network simulation function.

$\%$

$\%$ Generated by Neural Network Toolbox function genFunction, 13-May-2019 11:20:54.

$\%$

$\%[\mathrm{Y}]=\operatorname{net} 20 \mathrm{unit}(\mathrm{X}, \sim, \sim)$ takes these arguments:

$\%$

$\% \quad \mathrm{X}=1 \mathrm{xTS}$ cell, 1 inputs over TS timesteps

$\%$ Each $X\{1$, ts $\}=11 \times$ matrix, input $\# 1$ at timestep ts.

$\%$

$\%$ and returns:

$\% \quad \mathrm{Y}=1 \mathrm{xTS}$ cell of 1 outputs over TS timesteps.

$\%$ Each $Y\{1$, ts $\}=1 x Q$ matrix, output $\# 1$ at timestep ts.

$\%$

$\%$ where $\mathrm{Q}$ is number of samples (or series) and TS is the number of timesteps.

$\% \#$ ok $<*$ RPMT0 $>$

$\%=====$ NEURAL NETWORK CONSTANTS $====$ 
$\%$ Input 1

x1_step1.xoffset $=[-50 ; 1 ; 2 ; 10 ; 200 ; 193.15 ; 138.15 ; 2 ; 0.87 ; 2.9 ; 1]$;

x1_step1.gain $=$

[0.0133333333333333; $0.002002002002002 ; 0.0416666666666667 ; 0.00050125313283208 ; 1.000$ $100010001 \mathrm{e}-$

06;0.00251572327044025;0.00254598688816753;1;0.141542816702052;0.0205973223480947;

$0.263157894736842]$

x1_step1.ymin $=-1$;

$\%$ Layer 1

b1 $=[0.33610887392501448 ;-4.7446311457129573 ;-$

4.1448912223398988;0.64007788467452087;-0.81043098006805026;-

$6.5986703265553306 ; 1.3527261750333839 ;-$

$1.3249088366340713 ; 1.4481020041428845 ; 0.94742588014662465 ; 2.5971081633001103 ; 0.617$ 47868440606757;-3.8733228695425348;-

$3.6546810821327531 ; 1.6782489313931448 ; 2.072174553247168 ;-$

3.1351796280896829;5.4004940851699876;-3.8552205080896464;6.4138576428442375];

IW1_1 = [0.094408055584908362 $1.5959085990751014-0.058308675800376311$

$0.031589478460926468-0.79700095176339902-0.19346066918637431-1.4577262319076727$

0.511087138861761161 .04795529022287390 .50818686635181554 -

$0.77667982006889758 ; 0.15229999687766996-5.0643823063257241-0.56896291003195343$

$0.665788395409859680 .59687668696949037-0.24453537569294871 .7262868104024871$

$0.53589685098619666-0.013421498816881417-0.52946865185530656-$

$0.41627376413031386 ;-0.010565063675375401-3.79928999605256120 .37766121131288904$

-4.8156022318755634 4.713381626872204 -0.102980035072481450.1252036709489999 -

0.176559997366335820 .160255326539080890 .080976167089742243

$0.086156864954703052 ;-0.0236085414812914130 .013927618173635387$ -

$0.233418904855201484 .4300207405153698-1.33498324260831485 .7590203710599139$ -

$5.49794965478004280 .32229442011789977-5.78041083763274523 .8034120715425477$

$1.6041894069746818 ; 0.0076896511763034289-0.064611217685759073$ -

$0.0106391319308917590 .19247780439291373-0.0035487812670924055$ -

$1.35503473271360831 .17064135318067850 .4317653591973945-0.22245732632934237$ -

$0.94109242004021576-0.19616281268104757 ;-0.010180484259201752$ -

$0.041934071870805674-4.41722242841090564 .55761763866093350 .98741762687626855$ -

$0.0068299192472694769-0.182218951485969250 .023706721391618869$

$0.0136121887410268570 .016738137288543006-0.012070456194660522 ;-$

$0.214963072819440962 .04122266969546920 .1258371332743001-0.38312090018229045$ -

$4.6050601636965052-8.4553114824041842-1.8045374225548505-4.208993006900239$

$0.132853528557182661 .8212075932203309-1.4149848020194666 ; 0.22679735831022793$

$0.17540012205457514-0.49934965430373690 .0650970019238465562 .7257140402896276$

0.82635002924238010 .735239478350715241 .21148650612033481 .5849746703631649

$0.27163624021614335-2.3322804276528575 ;-0.495422245774238163 .1562376767920597$ -

$0.907207759429388364 .9947154072925004-0.491173864230238010 .32871782851701492$ -

$5.0201017598993145-0.53336824199451971-1.59366199683294151 .0054290827329957$ -

$0.44048217143638413 ;-0.0249712765198391410 .090999318568719414$ - 
$0.033529368481669465-0.650666238573301530 .0012630274115190115$ $0.904240603098345970 .904604289359586370 .45864580729214682-0.69236676369743089$ $0.19734323464246964-0.45306275082174235 ;-0.38352099401425621-0.33709543056362751$ $0.76384813343506441-0.15162793469328212-0.902435259242803660 .046362385206327773$ $-0.54736841062193298-1.88559534713742520 .189409224062695280 .10675797207313387$ $0.46329950686166965 ;-0.16622931007539032-0.164911012771905840 .47034946011768519$ $-0.051422941252620501-2.5401981561356313-1.1543135113592740 .095090377769774248-$ $1.1848659458473105-2.42062063537844592 .0208121167912556-0.081506182511399572$;$0.010030165154225511-0.042175292237884188-4.14548499417211861 .9780157356985499$ $0.97452787340432279-0.0070520928634246827-0.17468228085715373$ 0.0236751690539074850 .0115663728108375730 .01591778544157128 $0.01226001859060822 ;-0.060833031166098457-0.012592890817267119$ $0.0582868591640422760 .0157630831774998970 .57910642463859241-1.6284802376529615$ $7.25503384874985270 .66783607781025489-5.2087613092294474-3.9867253565901706$ $0.27362940525143764 ; 0.194193738148262071 .206949700187457-0.10118316210519752$ $0.079121871812447371 .9038087729890407-0.26753720633684402-2.6800138344336322$ $0.29531664813070119-0.175097366926515061 .0329365699000661-$ $0.62689450534809787 ; 0.019655590419467142-0.18982182192887928$ $0.0272721678691572220 .149987871177865252 .1056890761664806-1.5016127067480332$ $0.222520254423132-0.939212882465100930 .60977711448135608-1.1217002974167631$ $0.38271066110748825 ; 0.050489231795719389-0.081515101273949647$ $0.14149577663738075-3.1898892163608963-1.404956478934660 .041046472524860274$ $0.060909787123945347-0.139411144485873770 .16560773986003383$ $0.0199045394857991640 .31245723178170043 ; 0.129306168554846811 .0801637258803072$ $2.696257222391522713 .037484334219943-8.8579716768872352-0.064825391073574073$ 0.0159168395832072870 .00606390502696983830 .057869125203721693 $0.00183276916293780060 .18414833195890631 ;-0.011446134527076421-0.083024501366237$ $-0.16147230433089760 .0670369552323247520 .212350780081596860 .69335064699665638$ $0.196224199158614751 .5264241004488639-0.53662959892973183-2.0690147996953958$ $0.48549807185694177 ; 0.0164360072967088340 .358109276755673220 .22044644253969589$ $0.20471897402854106-4.43372913117016942 .85654174866440826 .9477056164441571$ 3.19121046425624493 .60231574438575654 .0467249609797724 -1.2786746039884396];

$\%$ Layer 2

b2 = -1.1652045360508585;

LW2_1 $=[-0.27577113943074594-0.22207542891099125-0.54217283969049146-$ $0.60452580786529897-1.383635804134914614 .3895070701548880 .064467565398804577$ 5.40483050808641790 .0546553409970666760 .646306123309222550 .91401821974243302 $5.409059390737049-14.9768583422413850 .497944554934597770 .34211290349182122$ $0.33078225906637754-0.571942084125638410 .129236109450175551 .4940287082461878$ $0.26758727577123126]$;

$\%$ Output 1

y1_step1.ymin $=-1$;

y1_step1.gain $=1.07413324997988$;

y1_step1.xoffset $=1.36965779066222$; 


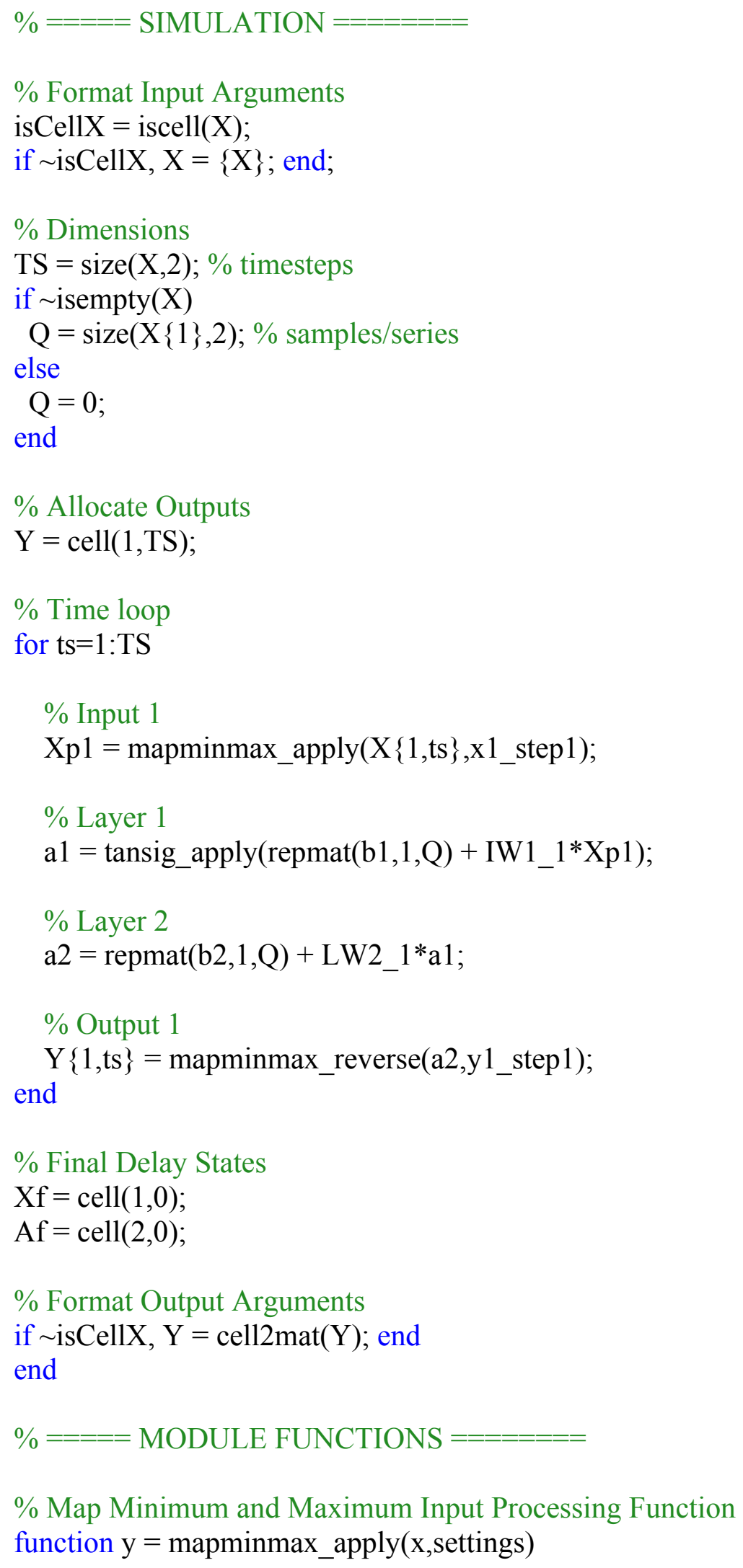




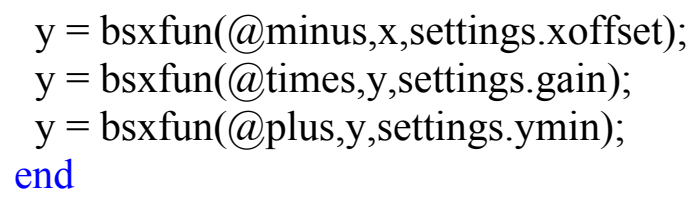

$\mathrm{y}=$ bsxfun(@minus,x,settings.xoffset);

$\mathrm{y}=$ bsxfun(@times,y,settings.gain);

y=bsxfun(@plus,y,settings.ymin);

end

\% Sigmoid Symmetric Transfer Function

function a $=$ tansig_apply $(\mathrm{n}, \sim)$

$\mathrm{a}=2 . /(1+\exp (-2 * n))-1$

end

\% Map Minimum and Maximum Output Reverse-Processing Function

function $\mathrm{x}=$ mapminmax_reverse(y,settings)

$\mathrm{x}=$ bsxfun(@minus,y,settings.ymin);

$\mathrm{x}=$ bsxfun(@rdivide,x,settings.gain);

$\mathrm{x}=$ bsxfun(@plus,x,settings.xoffset);

end 